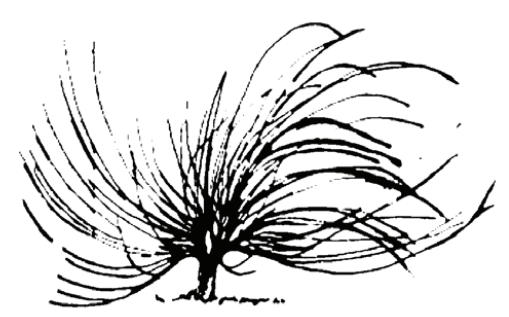

\title{
Aportes para una Revisión Bibliográfica Sobre la Teoría y Praxis del Currículo Educativo
}

\author{
Musa Ammar Majad Rondón ${ }^{l}$ \\ Universidad Alonso de Ojeda \\ San Cristóbal, Venezuela \\ musamajad@hotmail.com
}

\begin{abstract}
Resumen
El propósito de este artículo es lograr una aproximación, con miras hacia una revisión bibliográfica, de las conceptualizaciones de la teoría y praxis del currículo educativo, bien sea como modelo o como perfil curricular. Esto, debido a que se han propiciado múltiples líneas de pensamiento en torno a esta materia. Todo ello ha originado el desarrollo de diversas teorías que, en su conjunto, son trasunto de las distintas concepciones existentes al momento de abordar los elementos de la educación. En tal sentido, se efectuó una revisión documental y una interpretación de los textos bajo el paradigma crítico-reflexivo. Se establece que, del enfoque teórico emancipador del currículo y del enfoque del currículo basado en una reflexión sobre la práctica, se puede deducir una suerte de
\end{abstract}

\section{(ब) $(9 \Theta$}

Recibido: 11 de setiembre de 2015-Aprobado: 4 de diciembre de 2015

1 Licenciado en Letras (ULA). Magíster en Gerencia Educativa (UNET). Candidato a Doctor en Ciencias de la Educación (UBA). Contactos: web: http://musamajad.com.ve 
armonía, donde ambos presentan como elemento vinculante la dialéctica entre teoría y práctica.

Palabras clave: currículo, modelo curricular, perfil curricular

\begin{abstract}
The purpose of this article is to make an approximation to a literature review of the conceptualization of the theory and praxis of the educational curriculum, either as a model or as a curricular profile because multiple lines of thought on this matter have been fostered. All this has led to the development of various theories which, taken together, are a reflection of the different existing conceptions when addressing the elements of education. In this regard, a literature review and interpretation of texts under the critical - reflective paradigm was made. It is established that from the emancipatory-theoretical approach of the curriculum and the curriculum approach based on a reflection on the practice, a kind of harmony can be deduced, in which both have as a binding element a dialectic between theory and practice.
\end{abstract}

Key words: Curriculum, curriculum model, curriculum profile.

\title{
Consideraciones preliminares
}

Es un hecho conocido que el origen del término currículo se remonta a principios del siglo XVII. Fueron las universidades de Leiden y Glasgow, en el contexto de las reformas calvinistas, las que utilizaron el vocablo por vez primera. En ellas existe una “...noción de totalidad (ciclo completo) y de secuencia ordenada (la metáfora del progreso en una carrera de atletismo) de estudio" (Kemmis, 2008, p. 35). Por ende, se trata de una etapa inaugural donde la educación presenta un diseño pensado en cuanto a forma de organización e instrumento de eficiencia social.

Ahora bien, este término, de origen latino, es una expresión polisémica, multirreferente (recordando a Saussure), el cual, a partir de esta cualidad, conlleva a un amplio debate, por cuanto en él concurren 
múltiples concepciones sobre su significado y funciones. Se trata de una pluralidad conceptual. Al respecto, Tejada (2005) efectúa un análisis conceptual del currículo y establece los siguientes aspectos como relevantes: una evolución que lleva a valorarlo menos como un documento escrito para conseguir un título determinado y más como un proceso de diseño, desarrollo y evaluación; una mirada dirigida a una acción futu$r a$, siendo guía para la acción docente; un conjunto de conocimientos, procedimientos y aptitudes a adquirir; un programa cuyo escenario es lo educativo y sus factores o agentes; un análisis crítico-cultural de la educación como fenómeno; un paradigma particular de la época en el que se desarrolla.

Las conceptualizaciones del currículo educativo han propiciado múltiples líneas de pensamiento en torno a esta materia. Así, para Walker (2003) la teoría curricular refiere a un cuerpo de ideas coherente y sistemático que trata de aportar significado a los problemas y fenómenos propiamente curriculares. En este sentido, su objetivo final es "... establecer los fundamentos de la acción educativa y la práctica docente" (García, 1998, p. 102). Partiendo de esto, las teorías del currículo son de índole social. Esto, no solo porque reflejan la historia de las sociedades, sino también porque implican lo que concierne a su cambio y el papel preponderante de la educación en ellos. Así, en el informe presentado a la UNESCO por Delors (2001, p. 73), en representación de la Comisión Internacional para la Educación en el siglo XXI, se incluyeron cuatro competencias básicas: “... aprender a conocer, aprender a hacer, aprender a vivir con los demás y aprender a ser".

En virtud de estas breves reflexiones, el propósito de este artículo es lograr una aproximación, con miras hacia una revisión bibliográfica, de las conceptualizaciones de la teoría y praxis del currículo educativo, bien sea como modelo o como perfil curricular. Para ello, se realizará una revisión documental y una interpretación de los textos bajo el paradigma crítico-reflexivo.

\section{Espacios para las conceptualizaciones del currículo}

Si bien el origen del término currículo se ubica en los cenáculos universitarios europeos, como un campo de investigación y teorización de especificidad concreta, debe ubicarse en las primeras décadas del siglo pasado en Estados Unidos. Frente a este hecho, siguiendo a Salinas 
(1994), se pueden establecer dos eventos como referente. Primero, en 1918 se publica The Currículum, libro de Franklin Bobbitt; segundo, se da también la publicación de los Cardinal Principles of Secondary Education, de Kingsley.

Además de estos hitos, resalta también la publicación, en 1927, del XXVI Anuario de la Sociedad Nacional de Estudio de la Educación. En este último, en cuanto receptáculo de un fecundo intercambio de ideas, se observan dos posiciones completamente diferenciadas: por una parte, está el percibir al currículo como una necesidad cuyo fundamento se encuentra en los requerimientos de la sociedad; por otra parte, está el conjeturar que este mismo fundamento se haya en las parvedades del estudiante.

Aunado a lo anterior, según Beauchamp (2006), con el paso del tiempo se reconocen tres espacios de desarrollo para las investigaciones y teorizaciones del currículo: 1. Los estudios (académicos, investigadores, evaluadores); 2. Las prescripciones (estudiosos y la administración, como en sistemas educativos centralizados); y, 3. El desarrollo y ejecución práctica (profesores y estudiantes). Por ende, el currículo incluye aspectos de diseño, ingeniería, evaluación, teoría, investigación, entre otros.

De manera más descriptiva, se tiene entonces que:

...la teoría del currículum se ocupa de justificar la enseñanza intencional... lo que requiere plantearse qué contenidos se van a enseñar, por qué seleccionar esos contenidos y no otros, con qué criterios se seleccionan, al servicio de qué objetivos, con qué orden se enseñan, por medio de qué actividades, de qué agentes puede uno servirse, con qué normas se regulará el proceso, y cómo se comprobará si las decisiones tomadas son adecuadas o no. (Gimeno, 1983, p. 346)

Así entendida, la teoría del currículo tiene una misión inobjetable: organizar y guiar el desarrollo en la práctica del diseño o programación de la enseñanza que previamente se ha confeccionado, lo que supone establecer un puente entre los planteamientos de la teoría pedagógica y la fundamentación filosófica, sociológica, psicológica, epistemológica y la praxis de la enseñanza. Ello, pues “...el estudio del currículum se interesa por la relación entre sus dos acepciones: como intención y como realidad" (Stenhouse, 2012, p. 27). 
Esto no presupone que el currículo haya de ser concebido como una suerte de plan estático, que obvie las relaciones entre teoría y práctica curricular o educación y sociedad, pues no se trata de una figura, plan o cartografía que se elabora fuera de contexto. Al contrario, es modelado desde las franjas volitivas que le enmarcan: lo económico, social, cultural, político, religioso, en un momento histórico determinado. Por tanto, se descubre que los fundamentos que dan punto de inicio y basamento a la teoría curricular son epistémicos, psicológicos, filosóficos, sociológicos, entre otros.

\section{Enfoques curriculares teóricos}

Resulta evidente que esta multiplicidad de aproximaciones teóricas, se relaciona con las diversas funciones sociales atribuidas al hecho educativo. En este orden de ideas, siguiendo a Bolívar (2008), resulta pertinente mencionar los enfoques científico-técnico, deliberativo-práctico y socio-crítico.

Primeramente, el enfoque científico-técnico refiere a un modelo que conlleva la idea de explayar al currículo la ideología de la eficiencia social y utilitarista propia del mundo empresarial, suponiendo un primer intento de racionalizar los procesos de enseñanza. Sus momentos determinantes fueron las obras de Bobbitt y de Tyler, publicadas hacia 1918 y 1949, respectivamente. Se trata de un enfoque implícitamente ligado a una racionalidad técnica del currículo, propiciando que la planificación de este se centre en la racionalidad de hacer coexistir la dialogicidad medios y fines; es decir, implantar los productos o resultados de la instrucción con el objetivo de establecer los medios más eficaces para conseguirlos.

Ahora bien, en torno a la concepción más o menos técnica del currículo en Occidente, la idea del diseño, tan propia de las esferas administrativas, es importante. Se trata de una suerte de equilibrio direccional, que pretende no sólo guiar, sino también ayudar a los profesionales docentes. No obstante, es obvio que en este apartado el docente se limita a un accionar en cuanto emisor y ejecutor de una teoría o planteamiento curricular originada en la concepción de algún especialista en el área.

Así pues, es importante entonces lo señalado por Kemmis (2008, p. 73), para quien esta visión técnica “...relegaba a los encargados de 
confeccionar el currículum el papel de tecnólogos, dependientes de los científicos investigadores puros de la psicología, y convertía a los profesores en técnicos -operarios- que, a su vez, dependían de los tecnólogos". En este orden de ideas, Guarro (1999, pp. 119-121) indica que esta manera de idear la planificación y el proceso educativo implica unos efectos secundarios. Entre estos se encuentran: a. El hecho de separar lo educativo o curricular de lo instructivo, derivando en una dependencia absoluta de los docentes para con los especialistas; b. El cuestionar la participación de los involucrados al momento de considerar el proceso educativo, pues se le ve como una cuestión técnica y se le separa de sus dimensión social, pública o política; y, c. El establecer que los problemas curriculares deben abordarse desde el conocimiento teórico y los procedimientos que de este se derivan, anulando las características del contexto específico donde se suscitan.

En relación con el enfoque deliberativo-práctico, se trata de una perspectiva nueva apalancada en el pragmatismo filosófico y pedagógico de Dewey, en la epistemología genética de Piaget, en la psicología cognitiva aplicada a la educación y en la teoría del desarrollo moral de Kohlberg, tal y como indica Moreno (1999).

Asimismo, Schwab, hacia 1969, fue el primer autor que requirió la racionalidad deliberativa como alternativa a la racionalidad técnica. Se establecía una apertura del discurso técnico curricular a la esfera pragmática. Por ende, se concebía que las soluciones o respuestas únicas no fueran pertinentes; antes bien, en función del contexto, resulta imprescindible el consenso en torno a ellas.

Esta racionalidad deliberativa, rebautizada como racionalidad comunicativa, mantuvo una importante repercusión en las reformas educativas inglesas, sobre todo hacia los años setenta y ochenta del siglo pasado. Así, el currículo se transforma en "...una tentativa para comunicar los principios y rasgos esenciales de un propósito educativo, de forma tal que permanezca abierto a discusión crítica y pueda ser trasladado efectivamente a la práctica" (Stenhouse, 2012, p. 29).

Lo anterior implica idear en torno al currículo un proceso abierto a las reflexiones docentes junto a las investigaciones relacionadas con la praxis, construyendo y disponiendo de un “....conocimiento práctico sobre el propio proceso de diseño y desarrollo del currículum, sobre su articulación y su puesta en práctica" (Moreno, 1999, p.133). 
Como se observa, el enfoque deliberativo-práctico tiende a resultar más democratizado, lo que resulta de implicar al docente en el proceso de diseño y planificación del currículo. Además, tiene como ventaja que presupone un proceso de formación permanente desde la praxis. Ello fortalece el vínculo entre diseño, investigación y desarrollo profesional del docente. En líneas generales, el currículo aparece en cuanto "... proyecto en ejecución que se verifica en la acción del aula en la que los sujetos son parte del mismo" (Álvarez, 1987, p. 143).

Respecto a lo anterior, hacia los años ochenta, en el Reino Unido, indica Moreno (1999) que se originó la formulación de objetivos didácticos, no en términos de capacidades sino de conductas observables. También, se propiciaron propuestas de integración interdisciplinar, orientación cognitiva del currículo, regeneración de prácticas curriculares como la orientación y la tutoría, el desarrollo de actitudes democráticas y cívicas, entre otras.

Sin embargo, más allá de estas ventajas, el enfoque deliberativo-práctico puede contener algunas contradicciones. Sobre esto, Bolívar (2005) señala que el abuso de la racionalidad deliberativa puede llevar a que esta sea vista como algo indiscutible, concibiendo como positiva o viable cualquier tipo de interpretación bajo el hecho de que esta es de carácter personal e individual.

Finalmente, el enfoque socio-crítico tiene que ver con un movimiento de los años setenta del siglo pasado que buscaba una reconceptualización del currículo, más allá de los dos enfoques ya señalados. No solo se pretendía renovar la teoría curricular, sino que se intentaba realizar una crítica del sistema, bajo el manto de diversos enfoques filosóficos de tradición europea, como el neomarxismo, el existencialismo, la fenomenología, el psicoanálisis, la sociología de la educación y la teoría crítica de la Escuela de Fráncfort.

En este orden de ideas, el enfoque socio-crítico ubica la problemática curricular en una perspectiva histórica, lo que permea la posibilidad de establecer las relaciones entre la organización educativa y el entorno social, es decir, entre el conocimiento y el acceso a este por los disímiles grupos sociales. Aquí impera el razonamiento dialéctico, cuyos intereses son calificados por Habermas (2002/2004) como emancipadores. Esto significa propender a acciones que devengan en condiciones para transformar los vínculos y accesos sociales distorsionados, 
"...en donde las personas traten de superar la irracionalidad y la injusticia que desvirtúa sus vidas" (Kemmis, 2008, p. 92).

Por tanto, se critican las relaciones de poder, buscando un cambio social emancipador a partir de la educación, pues para Bolívar (2008) “...el conocimiento y experiencia escolar, como práctica social, están siempre, socialmente condicionadas. Se han de desvelar dichos condicionantes para que los actores tomen conciencia..." (p. 150).

En este sentido, Grundy (2010) establece una serie de principios que han de regir tal interés emancipador: ser una praxis sustentada por la reflexión, bajo las condiciones del contexto real en el que se desarrolla, manteniendo su cualidad de acto social, no circunscribiéndose solo al aprendizaje, donde los participantes tengan un rol activo en la construcción del saber y del significado de este como constructo social. Esto hace ver al docente, en cuanto investigador, como constructor activo del currículo, suscitando la participación crítico-reflexiva de los estudiantes, contribuyendo a la transformación social. Así, “....pretende, en relación con los sujetos, no sólo iluminar su pensamiento, sino también dotarle de un poder reflexivo y crítico para su inserción en un proceso más amplio de transformación social" (González y Escudero, 1987, p. 45).

\section{Relación con la praxis}

La importancia de los múltiples aportes teóricos en torno al currículo, más allá de su carga semántica, estriba en que ellos generan y realimentan un debate profundo y constante, no sólo de enfoques teóricos, sino también de prácticas educativas. Sobre esta producción teórica curricular, Kemmis (2008) indica que elementos importantes en la reconstrucción de la teoría social han sido las relaciones con la práctica. Esto es abordado por Stenhouse (2012) en cuanto comprensión del proceso de adquisición de conocimientos a través de la práctica. En resumen, se afianza la práctica de la enseñanza como bastión en las teorías curriculares. Así, ya Lundgren (2012) establece que el currículo, como concepto, viene determinado por un proceso de transición entre la sociedad y la educación, donde se imbrican explicaciones acerca de la puesta en práctica de la enseñanza.

En tal sentido, esta visión compartida entre Kemmis, Stenhouse, Lundgren ubica al currículo, junto con las tendencias que de él se derivan, en una perspectiva socio-crítica de las relaciones entre teoría y praxis. 
Esto se encuentra determinado por las plataformas culturales e históricas, siempre volitivas, de la educación y sus relaciones con la sociedad. Como praxis, la visión del currículo aportada por Grundy (2010) resulta importante. Ya el currículo no es solo un concepto, también es una construcción cultural que implica la organización idónea de un cuerpo de prácticas educativas humanas. Su basamento, por ende, es la ubicación del hombre, en cuanto ser, en cuanto talento humano, en el mundo.

Se trata, en consecuencia, de las personas dentro y fuera del ámbito educativo, porque el currículo se establece como elemento fundamental de la cultura y la sociedad en una realidad histórica específica. Así, Grundy (2010), apoyado en la teoría de los intereses constitutivos del conocimiento trazada por Habermas (2009), rehace, por así decirlo, no solo el sentido del currículo sino también la praxis de este; ello, basado en la acción del ser propiamente dicho: porque la manera en que se manifieste la acción del ser determinará lo que un grupo social especifico puede o no distinguir como conocimiento.

Siguiendo a Gimeno (2005), el enfoque que se tenga del currículo influye en la comprensión de la praxis educativa institucionalizada en su dimensión social. Por ende, el currículo no puede entenderse como un concepto estático. Se trata de un orden definido, que va desde los contenidos a desarrollar en el proceso educativo, pasando por los conocimientos a superar en un nivel o modalidad educativa determinada, hasta el conjunto de responsabilidades de las instituciones educativas para promover experiencias de aprendizajes.

Ahora bien, dichas perspectivas brindan legitimidad al currículo si se mantienen articuladas al sistema social y a la realidad pedagógica que alrededor de la enseñanza y el aprendizaje se han puesto de manifiesto a nivel histórico. En este orden de ideas, la función del currículo de la enseñanza obligatoria difiere del de una especialidad o modalidad universitaria y profesional, lo que tiene su trasunto en contenidos, formas y esquemas de racionalización interna diferentes.

Esto se debe a las distintas funciones sociales de cada nivel, además de lo “...peculiar [de] la realidad social y pedagógica que en torno [a] los mismos se ha generado históricamente" (Gimeno, 2002, p. 16). Así, la teoría curricular contribuye a la crítica y renovación no sólo en torno a las situaciones y condiciones en que se desarrolla el currículo, sino también en función de la reflexión sobre la praxis educativa. 
En el sentido del currículo como praxis, Álvarez (2001) subraya que este incluye la perspectiva de sus protagonistas, es decir, de aquellos que participan en la situación real del aula como espacio físico. Así, es viable entender que el currículo, como indica De Alba (2007), comporta en su praxis una serie de virtudes y características: es maleable, interpretable, flexible y abierto.

Lo anterior sucede en un punto en el que, como ya se señaló, se incluyen cuatro competencias básicas en el hecho educativo: aprender a conocer, aprender a hacer, aprender a vivir con los demás y aprender a ser. No obstante, estos núcleos fueron actualizados, y, así, se declaró su carácter axiológico a partir de tres valores fundamentales: “... aprender a vivir con los demás, dar vida a las comunidades propias y superar el marco de la modernidad" (Delors, 2001, p. 76).

Se trata de tres ejes imbricados con la praxis educativa, todos enmarcados en el contexto específico y particular que le da personalidad, en pro de franjas volitivas específicas (económicas, religiosas, sociales, culturales, políticas, entre otras). Esto, pues el aprender a vivir con los demás implica una dimensión en el debate sobre la justicia social; el dar vida a las comunidades propias significa interactuar con el entorno y las personas; el superar el marco de la modernidad presupone las condiciones de ruptura y continuidad, donde la llegada de la sociedad moderna no ha de ser incompatible con la conservación de los valores y las tradiciones.

En resumen, este componente axiológico refuerza, en la praxis educativa, el lugar que todo hombre, amparado en la teoría de la glocalidad, ocupa en el universo. Siguiendo a Gallart y Jacinto (2007), en vez de brindar conocimientos útiles para insertar en el mercado laboral, se busca otorgar herramientas para vivir. En vez de atender y promover la diversidad, se busca dotar de las competencias necesarias para acceder a las culturas literarias, artísticas, científicas, que caracterizan la sociedad actual. Ello involucra que, en la praxis, las instituciones educativas desarrollen competencias que tiendan a hacer más capaces a las personas ante el plano laboral, pero también más plenas, creativas, autónomas en el desarrollo personal. Esto, sin desatender las reformulaciones propias de cada contexto histórico-social. 


\section{Reflexiones finales}

Analizados, por tanto, el enfoque teórico emancipador del currículo y el enfoque del currículo basado en una reflexión sobre la práctica, se deduce una suerte de armonía, donde ambos presentan como elemento vinculante la dialéctica entre teoría y praxis, siempre por medio del pensamiento crítico-reflexivo y de la acción autónoma de los protagonistas de los procesos de enseñanza- aprendizaje.

Aquí, entonces, el problema radica en interrelacionar los contextos de las prácticas educativas y comprender el proceso de enseñanza desde perspectivas ideológicas, conceptuales y metodológicas diversas. Por ello, resulta oportuno imbricar la relación entre el currículo real y el currículo oculto, es decir, la integración de las perspectivas de los estudiantes que aprenden y experimentan la práctica y su dimensión oculta, que no se manifiesta en los objetivos y contenidos establecidos.

Esto predetermina un currículo ideal que abarca no sólo las actividades de enseñanza sino también todas las condiciones del ambiente en el que estas se desarrollan. Así, cuanto más complejo sea el currículo, más problemático, difícil y, seguramente, inconcreto será su posible diseño.

En este orden, la complejidad como contexto es un avance hacia posibilidades creativas en las prácticas educativas. Esto puede darse tanto desde el diseño como desde la planificación del currículo. Se trata de una complejidad ejercida por docentes, estudiantes e institución, acorde a las demandas sociales y al tiempo en el que estas se originan. Lógicamente, se relaciona la dimensión experiencial de dichos actores con la dimensión global y colectiva.

Por tanto, es posible referir que, en la convergencia de ambas dimensiones, la naturaleza de la esfera curricular ha de originarse en los retos sociales pendientes, taras que día a día siguen apareciendo en estadísticas de organizaciones sin fines de lucro o medios de información y comunicación: la pobreza, la crisis ambiental, el acceso al conocimiento, la democracia, la inclusión, los derechos humanos, los valores morales, el desarrollo del talento humano, entre otros. Se trata de que el currículo recobre el germen inicial de sus propósitos e intereses. 


\section{Referencias bibliográficas}

Álvarez, C. (2001). El Diseño Curricular. La Habana: Pueblo y Educación. Álvarez, J. (1987). Dos perspectivas contrapuestas sobre el currículum y su desarrollo. Revista de Educación, 8 (282), 131-151.

Beauchamp, G. (2006). Currículum Theory. Michigan: University of Michigan.

Bolívar, A. (2005). El conocimiento de la enseñanza. Madrid: Morata

Bolívar, A. (2008). Didáctica y currículum: de la modernidad a la postmodernidad. Archidona: Aljibe.

De Alba, A. (2007). Currículum: crisis, mito y perspectivas. Madrid: M y Dávila.

Delors, J. (2001). Saludo al Forum Europeo de Administradores de la Educación. Oviedo: A. Príncipe Felipe.

Gallart, M. y Jacinto, C. (2007). Competencias Laborales. Revista Educación Técnico y Profesional, 2 (4), 15-23.

García, M. (1998). Diseño, desarrollo e innovación del currículum. Córdoba: Servicio de Publicaciones de la Universidad de Córdoba.

Gimeno, J. (1983). Teoría Del currículum. En Diccionario de las Ciencias de la Educación. Santillana. Madrid, España. Tomo I: Pp. 341-373.

Gimeno, J. (2002). El currículum una reflexión sobre la práctica. Madrid: Morata.

Gimeno, J. (2005). Comprender y transformar la enseñanza. Madrid: Morata.

González, M. y Escudero, J. (1987). Innovación educativa: teoría y procesos de desarrollo. Barcelona: Humanitas.

Grundy, S. (2010). Producto o praxis del currículum. Madrid: Morata. Guarro, A. (1999). Modelos de diseño de corte técnico y experto: descripción y balance crítico. En Escudero, J. (Ed.). Diseño, desarrollo e innovación del currículum. Síntesis. Madrid, España. Pp. 99-122.

Habermas, J. (2002). Knowledge and human interests. London: Heinemann. Habermas, J. (2004). Theory and practice. London: Heinemann.

Habermas, J. (2009). Teoría de la acción comunicativa. Madrid: Altea, Taurus. Kemmis, S. (2008). El currículum: más allá de la teoría de la reproducción. Madrid: Morata.

Lundgren, U. (2012). Teoría del curriculum y escolarización. Madrid: Morata. 
Moreno, J. (1999). Modelos de corte deliberativo y práctico: descripción y balance. En Escudero, J. (Ed.). Diseño, desarrollo e innovación del currículum. Síntesis. Madrid, España. Pp. 123-144.

Salinas, D. (1994). La planificación de la enseñanza: ¿Técnica, sentido común o saber profesional? En Angulo, J. y Blanco, N. (Coords.) Teoría y desarrollo del currículum. Aljibe. Málaga, España. Pp. 72-89.

Stenhouse, L. (2012). Investigación y desarrollo del currículum. Madrid: Morata.

Tejada, J. (2005). Didáctica-Currículum. Diseño, desarrollo y evaluación curricular. Barcelona: Davinci Continental, S.L.

Walker, D. (2003). Fundamentals of curriculum: Passion and professionalism. Mahwah, N. J.: L. Erlbaum Associates. 